\title{
Nueva fórmula láctea: biodisponibilidad del hierro y efecto en la prevención de su deficiencia en lactantes
}

\author{
Eva Hertrampf D. ${ }^{1}$; Fernando Pizarro A. ; Adriana Pereyra L. ${ }^{1}$; \\ Virginia Vega B. ${ }^{1}$ \\ Iron bioavailability and effect on prevention of iron deficiency \\ of a modified cow's milk formula.
}

\begin{abstract}
Iron bioavailabiljty from a modified cow milk, formula (LPM) (which included additional iron as 5 mg; ferrous sulfate and ascorbic acid. $50 \mathrm{mg} / \mathrm{ll}$ ! was examined in 11 adult women using the extrinsic radioactive tag method. The geometric mean absorption from LPM was $13 \%$ (reierence dose absorption $=26 \%$ ). The eftect of this formula on iron nutrition of infants was studied in 43 healthy term infants weaned spontaneously before 3 months of age which were given LPY until 9 inonths of age. Another 45 infants (controls) were ted full fat, non fortified powdered cow's milk (LP) as delivered through the National Complernentary Food Program (PNAC-Chile) and aditional 45 infants were given fortified a cow's milk formula (LFI with ferrcus sulfate iiron: $15 \mathrm{mg} / /$ and ascorbic acid: $100 \mathrm{mg} / \mathrm{ll}$. Solid foods (vegetables arid meat) were introduced to all these babies from age 4 months. At 9 months of age $4 \%$ infants fod LPM has evidence of anemia $v$ s. $2 \%$ and $20 \%$ of infants given LF and LP formulae respectively. This results suggets that LPM is eftective in preventing iron deficiency anemia in infants.

(Key words: mcdified cow's milk formulae, iron deficiency, bioavailability.)
\end{abstract}

La anemia por deficiencia de hierro constituye el déficit nutricional más frecuente en la pobla. ción de lactantes chilenos. Estudios de prevalencia realizados en la población de lactantes beneficiarios del Servicio de Salud muestran repetidamente en los últimos 15 años una frecuencia de niños anémicos al año de edad que varía entre el 30 al $40 \%{ }^{1-3}$.

lnvestigaciones realizadas en nuestro país demuestran la factibilidad de erradicar la anemia por déficit de hierro, mediante la ingestión de leche de vaca adecuadamente fortificada con hierro ${ }^{4-6}$. Sin embargo, en el Programa Nacional de Alimentación Complementaria (PNAC, de Chile) aún no ha sido incluida la entrega de una leche fortificada con hierro en el primer año de vida. Este trabajo forma parte de la evaluación integral de una leche de vaca en polvo modifica. da (LPM) formulada para el lactante ${ }^{7-9}$. El objetivo fue medir la biodisponibilidad del hierro de esta leche y su efecto en la nutrición de hierro de los lactantes.

1. INTA, Instituto de Nutrición y Tecnología de los Alimentos, Universidad de Chile.

Financiado por Ministerio de Salud.

\section{Material y Métodos}

Composición de lo fórmula. Corresponde a una leche adaptada a los requerimientos del grupo de lactantes menores de 1 año, diseñada con el objetivo de reemplazar la leche de vaca entera actualmente entregada a traves del PNAC. Esta fórmula se caracteriza por dilución de las ptoteínas y del Na, K y Cl, aumento del aporte de ácidos grasos esenciales (linoleico); la adición de maltodextrinas para mejorar ka digestibitidad, y la adición de vitaminas y minerales: ácido ascótbico $50 \mathrm{mg} / 1$; hietro $=7 \mathrm{mg} / \mathrm{l}$ en forma de sulfato fertoso y zinc $5 \mathrm{mg} \cdot 1$. Cabe destacar que la partida de lech empleada en este estudio resultó con un contenido menor al for. mulado $(5 \mathrm{mg} / 1)$.

Estudio de absorción de hierro. Once mujeres multíparas, aparentemente sanas, con edades entre 35 a 45 afios, participaron como voluntarias en este estudio previa obtención del consentimiento escrito de cada voluntaria. El protocolo fue aprobado por el Comité Etico Institucional, siendo, a su vez, aprobadas las dosis de radiactividaó por la Comisión Chilena de Energía Nuclear.

Procedimientos. Se midió la absorción del hierro de la LPM y de una dosis de referencia de ascorbato ferroso mediante una têcruca doble isotópica $\left(\mathrm{Fe}^{53}\right.$ y $\mathrm{Fe}^{59}$ ). El uso de la dosis de referencia permite comparar los resultados entre distintos estudios. Las bebidas fueron consumidas en mañanas consecutivas en estado de 
ayunas, no permitiéndose la ingesta de alimentos, excepto agua, durante las siguientes 4 horas. El dia 1 recibieron 250 ml de LPM reconstituida al $15 \%$, mar* cada extrínsecamente con 1 uCi de ${ }^{55} \mathrm{FeCl}_{3}$ (New England Nuclear, Boston, Mass.) y el dia 2 se les administró $150 \mathrm{ml}$ de una solución acuosa de ascorbato ferroso que conten ja $3 \mathrm{mg}$ de hiero como sulfato ferroso en una relación molar de $2: 1$ áido ascórbico/hierro y marcada con 3 uCi de ${ }^{59} \mathrm{EeSO}$, (New England Nuclear, Boston, Mass.). El día 15 se obtuvjer on muestras de sangre venosa para medir la radiactividad incorporada en los eritrocitos. La radiactividad ingerida se calculó en baste a duplicados de $10 \mathrm{ml} \mathrm{de} \mathrm{sngre} \mathrm{de} \mathrm{los} \mathrm{sujetos}$ $y$ en alícuotas en triplicado de las bebidas ingeridas para el recuento diferencial de ${ }^{5}$ Fe y ${ }^{39}$ Fe a través dol método de Lakins y Brown ${ }^{10}$. La actividad de los radioisótopos en las muestras procesadas fue determínada usando un espectrómetro (Nuclear Clicago, Chicago, III). Los valores de absorción fueron calculados asumiendo que el $90 \%$ de la radiactividad absorbida estaba presente en la hemoglobina de los eritrocitos circulantes. El volumen sanguíneo de cada sujeto fue estimado en base al sexo, cl peso y la talla.

Estudio longitudints. Tres grupos de lactantes fueron seguidos, desde los 3 a los 9 meses de vida, en el Consultorio Santa Julia del Area Oriente de la ciudad de Santjago, dependiente del Ministerio de Salud. Se seleccionaron lactantes con un peso de nacimiento mayor de $2.500 \mathrm{~g}$, sin anormalidades congénitas, desnutrición o complicaciones perinatales y que no hubiesen recibido hietro medicina] o transfusiones sanguineas. Fllo debíun haber sido parcial o totalmente destetados antes de los 3 meses de edad, siendo asignados al azar a los siguientes gru. pos:

LPM: 43 lactantes, que recibieron la leche en estudio, envasada en bolsa de poljetileno y caja de cartón ( $\mathrm{Fe}=5 \mathrm{mg} / 100 \mathrm{~g}$ ).

Leche Fortificada (LF): 45 lactantes, quienes recibieron una leche entera en polvo ( $26 \%$ de materia gra. sa) con $15 \mathrm{mg}$ de hierro (en forma de sulfato fer roso) y $100 \mathrm{mg}$ de ácido ascósbico/100 g de polvo. Envasada al vacio en tarros de hojalata.

Leche Programática (LP): 45 lactantes que recibie. ton la leche entera actualmente distribuida por al PNAC, la cual no está tortificada con hierro ni ácido ascórbioo.

Las madres fueron instruidas para preparar la LPM a) $15 \%$; la LF y la LP en djlución al 10\% con agregados de sacarosa al $5 \%$ y harina de maíz al 3\%. La indicación sobre la introducción de alimentos sólidos fue similar en los grupos, consistiendo en la ingestión de frutas a $\operatorname{los} 3$ meses, sopa de carne y vegetales a los 4 meses, legumbres y huevos a los 6 meses y la alimen. tación regular del hogar a los 9 meses.

El control de niño sano fue realizado por los pediatras autores de este trabajo, mensualmente, desde los 3 a los 9 meses. Cada control clínico incluyó un examen físico, una evaluación de los desarrollos antropométrico y psiconotor e instrucciones sobre alimentacjón e inmunizaciones. Ia motbilidad fue atendida por el mismo grupo de médicos.

Los sujetos fueron visitados en su domicilio semanalmente por enfermeras universitarias del equipo de investigadores, desde $\operatorname{los} 3$ a los 9 meses de edad.
En cada visita se realizó educación sobre puericultura; una encuesta alimentaria por el método de tecordatorio de 24 horas, con la firalidad de verificar la ingesta de leche y cuantificar el aporte de hierfo proveniente del resto de la dieta; una encuesta de morbilidad para conocer ell número y duración de los episodios de infecciones respiratorias y de diarreas. Además, a los 9 meses de edad se realizó una encuesta socioeconómica por el método de Graffar modificado.

A los 9 meses de edad se obturo una muestra de sangre venosa en ayunas para medir el lenatocrito, la hemoglobina (Hb), volumen corpuscular medio (VCM), (Coulter Counter, ZBI Hialeah, F ), hjerro sérico (Fe) y capacidad total de combinación de la transferrina $(T I B C)^{11}$, protoporfirina libre eritrocitaria (PEL) (hematolluorimetro AVIV, Lakewood, NJ) y ferritina sérica (FS) (Gamma Dab 125 I Radioimmunoassay, Travenol, Cambridge, Ma).

E] protocolo de este estudio fue aprobado por el Comité de Etica Institucional. El consentimiento informado de los padres se obtuvo previo al ingreso de los lactantes al protocolo.

Andisis de los datos: Se definió como valores anormales de $\mathrm{Hb}<11 \mathrm{~g} / \mathrm{dl}$ (etiterio OMS), oturación de la transferrina (Sat) $<10 \%$, PFL $>120 \mathrm{ug} / \mathrm{dl}$ de GR $y$ FS $<10 \mathrm{ug} / \mathrm{L}$. En el análisis estadistico se utilizaron el chj cuadrado y la prueba de t de Student.

\section{Resultados}

Estudio de absorción. La absorción de hierro de la LPM fue alta, siendo el promedio del grupo de $13,1 \%$. La absorción de hierro de la dosis de referencia $(26,2 \%)$ sugiere que el grupo en el cual se realizó la medición presenta una nutrición de hierro caracterizada por depósitos leve. mente depletados (tabla 1).

Estudio longitudinal. Las causas de deserción de los sujetos fueron similares en los grupos y no estuvieron relacionadas con la aceptabilidad de b leche. Los tres grupos resultaron homogéneos en peso de racimiento, composición por sexo, crecimiento, morbilidad intercurrente $y$ nivel socioeconómico.

La aceptabilidad de las tres leches fue excelente. En los tres grupos la ingesta de hierro dietario, excluido el hierro de fortificación de la leche, fue simitar y muy escasa.

Al comparar la LPM con la LP a los 9 meses de edad, los promedios de los parámetros de nutrición de hierro mostraron diferencias favorabłes al grupo que recibió la LPM, siendo éstas estadisticamente significativas en la $\mathrm{Hb}$ (p $<0,005)$ y en la Sat $(\mathrm{p}<0,0 \mathrm{l})$. Las diferencias de los valores del grupo L.PM en relación al gripo control óptimo (LF) no fueron significativas (tabla 2). El porcentaje de sujetos anémicos fue sustancialmente más bajo en el grupo LPM que 
Tabla I

Absorciön de hierro de la LPM

\begin{tabular}{ccc}
\hline Sujeros & $\begin{array}{c}\text { Absorción de hierro (\% de la đosis) } \\
\text { LPM }\end{array}$ & $\begin{array}{c}\text { Ascorbato } \\
\text { ferroso }\end{array}$ \\
\hline 1. & 20,45 & 59,40 \\
2. & $\mathbf{4 2 , 7 2}$ & 57,42 \\
3. & 21,24 & 46,38 \\
4. & 15,67 & 41,14 \\
5. & 9,24 & 35,44 \\
6. & 23,54 & 31,53 \\
7. & 15,44 & 28,37 \\
8. & 4,72 & 13,02 \\
9. & 8,24 & 12,47 \\
10. & 7,63 & 11,73 \\
11. & 6,52 & 10,01 \\
Promedio* & 13,06 & 26,17 \\
DE* & $6,94-24,57$ & $13,75-49,81$ \\
\hline
\end{tabular}

* Promedio geométrico y rango de $1 \mathrm{DE}$.

en el grupo LP y similar al grupo LF; sin em. bargo, la cantidad de hjerro aportada por la LPM resultó insuficiente para obtener depósitos adecuadamente depletados (figura 1).

\section{Discusión}

El lactante presenta una dependencia desproporcionada de fuentes externas de hierro comparado con el adulto, hecho que explica la alta prevalencia encontrada a esta edad ${ }^{12}$.

Varios estudios han demostrado la efectividad de leches especialmente adaptada para lactantes en la prevención de la deficiencia de hierro ${ }^{13-15}$. Sin embargo, la tecnologia empleada en estos productos determina costos altos, que los aleja del consumo masivo en sociedades pobres. En base a estos antecedentes, en nuestro país hemos demostrado la factibilidad de erradicar la anemia por deficiencia de hierro en el lactante a través de leches de vaca fortificadas ${ }^{4-6}$. Sin embargo,

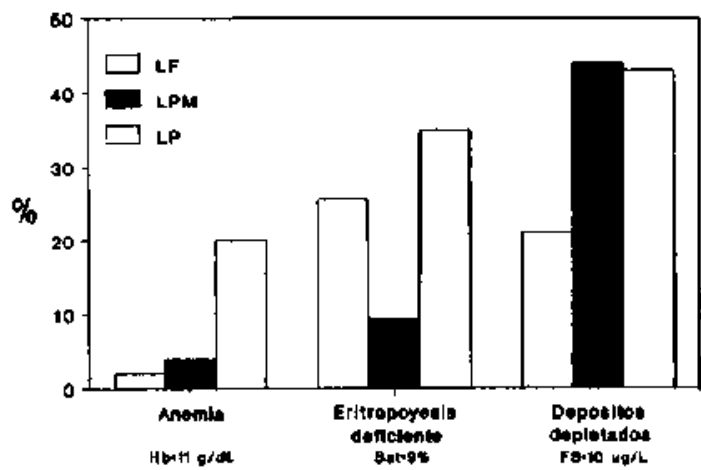

Fig. 1: Porcentaje de los lactantes deficientes en hierro a los 9 meses de edad.

estos productos, por sus altos contenidos de hierro, han requerido de envases en tarros de hojalata impermeables al oxígeno, debido a la gran capacidad de las sales de Fe de promover rancjdez de las grasas. Este hecho constituye un factor limitante cuando se considera el costo de la leche necesaria para un programa de entrega masiva, como el de Chile. En este caso se ha calculado que el cambio de una leche no fortificada a una fortificada implicaría un aumento del costo de cerca del 14\%. De este porcentaje, cerca del 1\% reside en la adición de sulfato ferroso y ácido ascórbico y $13 \%$ en el cambio de envase

Este estudio demuestra que el consumo de la LPM es capaz de prevenir la anemia por defjciencia de hierro en la pablación lactante de nuestro país. El estudio de absorción demostró que la biodisponibilidad del hierro de este producto es sorprendentemente alta, de un $20 \%$ al referir el promedio de absorción encontrado a $40 \%$ de absorción de la dosis de referencia ${ }^{16}$ (valor aceptado como propio de una población con depósitos depletados de hierro, como es el caso de los lactantes). De tal modo que el consumo diario de $700 \mathrm{mi}$, aportando casi $1 \mathrm{mg}$ de hierro absorbido, cubriría los requerimientos

Tabla 2

Nutrición de hierro de lactantes a los 9 meses de edad

\begin{tabular}{lcccrr}
\hline Grupo & $\mathbf{n}$ & $\begin{array}{c}\mathrm{Hb} \\
(\mathrm{g} / \mathrm{dL})\end{array}$ & $\begin{array}{c}\text { Sat } \\
(\%)\end{array}$ & $\begin{array}{c}\text { PEL } \\
(\mathrm{ug} / \mathrm{dL} \text { GR })\end{array}$ & $\begin{array}{c}\text { FS } \\
(\mathrm{ug} / \mathrm{L})^{*}\end{array}$ \\
\hline LF & 45 & $12,64 \pm 0,85$ & $14,6 \pm 6,4$ & $99 \pm 25$ & $15(8-27)$ \\
LPM & 43 & $12,23 \pm 1,09$ & $15,1 \pm 7,2$ & $88 \pm 30$ & $12(7-20)$ \\
LP & 45 & $11,88 \pm 1,15$ & $12,3 \pm 7,0$ & $129 \pm 68$ & $10(5-18)$ \\
\hline
\end{tabular}

* Promedio geométrico y rango de $1 \mathrm{DE}$. 
de hierro de la mayoría de los lactantes. Esta biodisponibilidad alta puede deberse a factores propios de la formulación de esta leche, tales como la concentración de ácido ascórbico ${ }^{17}$, la disminución del contenido de prote únas y fosfatos ${ }^{18}$ y la disminución del contenido de hierro que determina, en este caso, una óptima relación hierro/zinc ${ }^{19}$.

El cálculo teórico, recién mencionado, fue corroborado en este estudio, ya que là LPM logró prevenir la aparición de la anemia en la mayoría de los lactantes, siendo tan efectiva como la LF. Sin embargo, la disminución accidental del contenido de hierro de la partida empleada en este estudio se ve reflejada en el alto porcentaje de lactantes con depósitos de hierro depletados. Cabe destacar que la disminución del contenido de hierro de 15 a $7 \mathrm{mg} / \mathrm{l}$ permite el empleo de los mismos envases actua. les $^{7}$, aumentando la factibilidad de incorporar el producto en el PNAC.

Recientemente ha sido demostrado que hasta en casos con anemia leve por carencia de hierro aparecen déficit psicomotores a los 12 meses de edad $^{20,21}$. Más aún, resultados preliminares de un estudio de Walter y cols, en nuestro medio, muestran que estos lactantes cuya anemia fue corregida con tratamierito de hierro entre los 12 y 15 meses de edad presentan a los 5 años puntajes inferiores en exámenes de funciones intelectuales que aquellos que tenían una nutrición normal de hierro a los 12 meses de vida ${ }^{22}$. Dado que los lactantes raramente desarrollan anemia antes de los 6 meses, bastaría un periodo breve de deficiencia de hierro leve entre los 6 y los 12 meses de vida para producir secuelas tanto a nivel conductual como de rendimiento escolar. Estos hallazgos constituyen una justificación aún más poderosa para prevenir la deficiencia de hierro en el lactante.

\section{Resumen}

Ia biodisponibilidad del hierro de una leche en polvo adaptada especialmente para lactantes (LPM), que contiene hierro en forma de sulfato ferroso $(5 \mathrm{mg} / 1)$ y ácido ascórbico $(50 \mathrm{mg} / \mathrm{l})$, fue medida en 11 mujeres adultas, mediante el método doble isotópico. E1 promedio geométrico de absorción fue de 13\% (absorción de la do. sis de referencia: $26 \%$ ). El efecto de esta leche en la nutrición de hierro de lactantes se estudió en 43 sujetos de tétmino, sanos, destetados espontáneamente antes de los 3 meses de edad, los cuales recibieron LPM hasta los 9 meses de edad. Este grupo fue comparado con 45 lactantes alimentados con la leche de vaca entera, no fortificada (LP), que es entregada a través del Programa Nacional de Alimentación Complementaria (PNAC), y con 45 alimentados con una leche de vaca entera fortificada con sulfato ferroso (hierro: $15 \mathrm{mg} / \mathrm{t}$; ácido ascórbico 100 $\mathrm{mg} / 1)$. Todos los lactantes recibieron alimentos sólidos (carne y vegetales) desde los 4 meses de edad. A los 9 meses, cuando la nutrición de hierro fue evaluada, $4 \%$ de los lactantes que recibieron LPM tenja anemia vs. 2 y $20 \%$ de los lactantes alimentados con LF y LP, respectivamente. Este estudio demuestra que la LPM es efectiva en prevenir la anemia por deficiencia de hierro en el lactante.

(Palabras clave: hierso, biodisponibilidad, le. che de vaca modificada, fómula láctea para niños.)

\section{Referencias}

1. International Nutritional Anemia Consulfative Group: The prevalence of aneria in the world. Washington, D.C.: WHO Report. The Nuttition Foundation. 1985.

2. Rios, E.; Olivares, M.; Amar, $M$, et al.: Evaluation of irons status and prevalence of iron deficiency in infants in Chile. In: Underwood, B.A., ed. Nutrition Intervention Strategies in National Development. New York: Academic Press, 1983; 273 283.

3. International Nutritional Anemia Consultative Group: Combating iron deficiency in Chile: A case study. Washington, D.C.: WHO Report. The Nutrition Foundation, 1986.

4. Olivares, M.; Walter. T.: Hertrampl. E., ef al.. Prevention of iron deficiency by mil fortification. Acta Paediutr Scand 1989; Supp1 361: 103-108.

5. Walter, $T$.: Olivares, M.; Heytrampf, E.: Field trials of food fortification with iron: The experience in Chiłe. In: Lonnerdal, B., ed. Iron Metabilism in Infants. Boca Ratón, Florida: CRC Ptess [nc., 1990; 127-154.

6. Stekel, A.: Olivares, M.: Pizarro, F., et al.: Prevention of iron deficiency in infonts by milk fortification. l. A field trial with a low fat milk. Arch Latinoamer Nutr 1986; 36:654-66I.

7. King, J.; De Pablo, S.; Montes de Oca, F.: Uauy. $R$ : Fornulación y evaluación de una fórmula infantil para el Programa Nacional de Alimentación Complementaria en Chile. Rev Chil Pediatr 1991. En prensa.

8. Casrillo, C.; Barrero, G.: Gatıás. Y.: Riumalló,

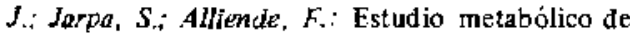
una nueva fórmula intanti diseñada para el Programa Nacional de Alinentación Complementaria. I. Balance nitrogenado y calórj̀co. Rev Chil Pediatr 1991. En prenso. 
9. Jury', G.i Castillo, C.; Atolah, E.; Puentes, R.:

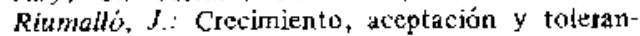
cia con una nueva fórmula láctea. Rev Chil Pediatr 1991. En prensa.

10. Eakins. I.; Brown, D.: An improved method for the simultaneous determination of ${ }^{55}[\mathrm{Ce}$ in blood by liquid scintillation counting Int J Appl Radiat 1sot $1966 ; 17: 391-397$.

11. Fisther, D.S.: Price, D.C.: A simple serum iron method using the new sensitjve chromagen tripiridyl-s-triazine. Clin Chem 1964; $10: 21-30$.

12. Dallmar, P.R.: Siimes, M.A.; Stekel, A.: lron deficiency in infancy and childhood. Am $J$ Clin Nutr 1980:33:86-118.

13. Warsh, A.: Long, H.: Stierwalt, R.N.: Comparative hematologic response to iron fortification of a milk formula for infants. Pediatrics 1959; 14: 404-412.

14. Sarimen. U.M; Sïmes, M.A.: lron absorption from infant nilk formula and the optimal level of iron supplementation. Acta Paediatr Scand 19?7:66: 719-722.

15. Hertrampt, E.: Cayazzo, M.: Pizarro, F.; Stekel, A.: Bioavailability of iron in soy-based tormula and its tiffect on iron nutriture in infancy. Pediatries $1986: 78: 640-645$

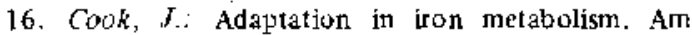
J Clin Nutr 1990:51:301-308.

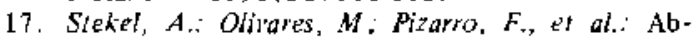
sorption of fortification iron from nilk formulas in infants. Am J. Clin Nutr 1986;43: 917-922.

18. Gross. $E_{\text {.: }}$ The relationships between milk protein and iron content on hematologic values in iniancy. J. Pediatr 1968; 73:521-530.

19. Solomons, $N_{*}-J_{a c i b}, R .:$ Studies on the bioavailability of zine in humans: et fects ol heme and nonlreme iron on the absorption of zinc. Am J Clin Nutr $1981 ; 34: 475-48$ 2.

20. Walter. T.: De Andra'a, 1.: Chodud, P.: Perales, $C_{.} G_{*}:$ Iron deficiency anemia: Adverse effects on infant psychomotor development. Pediatrics 1989:84: 7-17.

21. Lozoff. B.: Britenham. G.M.: Wolt A,W., et al.: Iron deficiency anemia and iron therapy affects on infant developmental test perlomance. Pediatrics 1987:79: $981-995$.

22. Hialter, $T$. De Andrace, I.: Castillo, $M$, et at.: Cognitive effects at 5 years in infants who were anemic at 12 months. Pediatr Res, abstr. En prens. 